\title{
Lissencephaly with cerebellar hypoplasia type D
}

INSERM

\section{Source}

INSERM. (1999). Orphanet: an online rare disease and orphan drug data base.

Lissencephaly with cerebellar hypoplasia type D. ORPHA:100014

Lissencephaly with cerebellar hypoplasia type $D(L C H d)$ is a form of lissencephaly with cerebellar hypoplasia characterized by pronounced microcephaly (at least \pm 3 SD), intellectual disability, spastic diplegia and moderate to severe cerebellar hypoplasia involving both vermis and hemispheres. 